\title{
Importance de l'association de cultures face aux variabilités climatiques dans les collines au Benin
}

\author{
HOUNDETE Codjo Jacques ${ }^{1}$., ASSONGBA Yédjanlognon Faustin ${ }^{182 .}$., YOKA Joseph ${ }^{3}$ \& DJEGO Julien \\ Gaudence ${ }^{2}$ \\ 1 Ecole Nationale Supérieure des Biosciences et Biotechnologies Appliquée (ENSBBA), Université Nationale, des \\ Sciences, Technologies, Ingénierie et Mathématiques (UNSTIM) Abomey Email : yedjanlognon@yahoo.fr I \\ yedjanlognon@gmail.com \\ ${ }^{2}$ Laboratoire d'Ecologie Appliquée, Faculté des Sciences Agronomiques, Université d'Abomey-Calavi, Cotonou, \\ Bénin. Email : gdjego@yahoo.fr \\ 3 Laboratoire de Botanique et Ecologie, Faculté des Sciences et Techniques, Université Marien Ngouabi, Brazzaville, \\ Congo. Email : joseph_yoka@yahoo.fr \\ Auteur correspondant : yedjanlognon@yahoo.fr / yedjanlognon@gmail.com
}

Original submitted in on $2^{\text {nd }}$ March 2020. Published online at www.m.elewa.org/journals/ on $30^{\text {th }}$ June 2020

https://doi.org/10.35759/JABs.150.3

\section{RÉSUMÉ}

Objectif: Cette étude vise à estimer les performances agronomiques de la production du mais par association à une espèce de la famille des cucurbitacaea «goussi» en vue de l'exploitation des processus écologiques.

Méthodologie et résultats : les essais ont été installés en champs paysans sur un sol ferrugineux tropical dans deux (2) villages de la commune de Glazoué. Le dispositif expérimental est en blocs aléatoires complets (BAC) avec deux répétitions ou blocs. Chaque bloc est subdivisé en deux parcelles élémentaires abritant chacune un traitement. Les traitements sont du maïs en culture pure (Témoin absolu T0) et maïs en association avec goussi (Test T1). Les données climatiques, agronomiques et de rentabilité financière ont été collectées sur cette expérimentation. En matière de résultat, il ressort que l'humidité au niveau des parcelles du système de l'association de maïs et goussi est supérieure à celle des parcelles du système de culture pure de maïs et ceci durant tous les temps de collectes. Le poids des épis était le plus élevé pour l'association maïs et goussi de même que pour tous les autres paramètres (le nombre de rangées par épis, le nombre de grains par épis et le poids de 100 graines).

Conclusion et application des résultats: la pratique agricole qui constitue à associer deux espèces végétales domestiquées Zea mays $L$. de la famille des Poaceaes et Citrullus lanatus (Thunb.) de la famille des Cucurbitaceae est une des meilleures stratégies mises en place par les producteurs pour les impacts des changements climatiques.

Mots clés : Mélange de culture ; variabilité climatique ; maïs-goussi ; rentabilité et Citrullus lanatus 


\title{
Importance of the association of crops facing climate variability in the hills in Benin
}

\begin{abstract}
Objective: This study aims to estimate the agronomic performance of corn production by association with a species of the cucurbitacaea family "goussi" for the exploitation of ecological processes.

Methodology and results: the trials were set up in peasant fields on tropical ferruginous soil in two (2) villages in the commune of Glazoué. The experimental setup is in complete random blocks (BAC) with two repetitions or blocks. Each block is subdivided into two elementary plots, each housing a treatment. The treatments are corn in pure culture (absolute control T0) and corn in association with goussi (Test T1). Climatic, agronomic and financial profitability data were collected on this experiment. In terms of results, it appears that the humidity in the plots of the system of the association of maize and goussi is higher than that of the plots of the system of pure cultivation of corn and this during all the times of collections. The weight of the cobs was highest for the corn and goussi combination as well as for all the other parameters (the number of rows per cob, the number of grains per cob and the weight of 100 seeds).

Conclusion and application of the results: the agricultural practice, which consists in associating two domesticated plant species Zea mays L. of the family Poaceae and Citrullus lanatus (Thunb.) of the family Cucurbitaceae is one of the best strategies implemented by producers to the impacts of climate change.

Keywords: Mixed cropping; climate variability; Maize-goussi; profitability and Citrullus lanatus
\end{abstract}

\section{INTRODUCTION}

Au Bénin, l'agriculture est la principale activité et occupant presque toute la population active (Assongba, 2014). Cette agriculture béninoise est marquée par une faible productivité en raison de son caractère extensif et les aléas climatiques. Le système de gestion des terres agricoles au Bénin est une alternance des cultures extensives et une mise en jachère sur de longues périodes. La variabilité climatique se manifeste essentiellement par la hausse de la température et la diminution des précipitations (Gnangle et al., 2012). Cette variabilité des précipitations accroit considérablement le risque de sécheresse (De Vries et al., 2011; Assongba, 2014). Les aléas climatiques, tant redoutés des agriculteurs de par son caractère aléatoire et la variation des précipitations constituent une contrainte majeure au développement des cultures pluviales. Ainsi, beaucoup d'agriculteurs hésitent, par précaution, à investir dans des cultures stratégiques comme les cultures vivrières, dont le rendement dépend pour une large part de la composante climatique. De plus, le déficit hydrique engendré par la sécheresse augmente la sensibilité des espèces aux valeurs extrêmes de température, notamment en dehors de la saison humide (Guyot, 1993). L'impact de cette modification du climat est d'autant plus fort sur l'agriculture familiale qui subit des mutations de l'environnement (Visoh et Assongba, 2017) ceci par la dégradation de la fertilité des sols, déforestation, l'érosion de la biodiversité avec comme corollaire la réduction de la durée de la période de végétation (Assongba, 2014). Le Bénin étant un pays cotonnier, le système de culture continue de coton et maïs sur des sols pauvres, sont des facteurs qui maximisent les risques de baisse de la fertilité des sols. Cela induit comme conséquences la baisse des rendements agricoles, la baisse des revenus et plus grave la fragilisation de la sécurité alimentaire. Ce système de culture a pour conséquences, la détérioration des systèmes de production, l'épuisement des sols aggravés par la forte croissance démographique. Ainsi, on assiste à un système de culture caractérisé par le travail intensif du sol, forte utilisation de pesticides et engrais chimiques, la vaine pâture et le brûlis des résidus de culture même système de culture en BurinaFaso souligné par Coulibaly et al., (2012c). Ce système de culture à base de cotonnier et de céréales ne permet pas de maintenir la fertilité des sols (Bado, 2002). Le Bénin possède une biodiversité d'espèces cultivables locales qui s'adaptent aux affres du changement climatique. 
En effet les variabilités climatiques qui se traduisent par plusieurs facteurs (décalage du calendrier climatique, retard des pluies, changement des hauteurs d'eau reçues annuellement, sécheresse plus marquée ou plus fréquente, apparition des phénomènes paroxystiques, évènements anormaux, températures variables tant temporelle et spatiale) modifient les conditions de production chez les végétaux (Dugué et al., 2012). Les Dès lors, les systèmes de culture sont devenus non adaptés et ne satisfaits plus les demandes en production (Morris et al., 2007 ; World Bank, 2009). Face à cette situation, les agriculteurs ont tendance à réduire les superficies cultivables d'une part et le nombre d'espèces ou de variétés cultivées d'autre part (Van Dusen et Taylor, 2005). Cette mesure prise se remarque beaucoup plus au Bénin sur les cultures céréalières en particulier le maïs. De ce fait, il y a des conséquences importantes sur le régime alimentaire des ménages agricoles ce qui influence la sécurité alimentaire. II y a donc un besoin réel de trouver de façon imminente des solutions pouvant garantir l'accessibilité et la disponibilité constante du maïs surtout en milieux ruraux. Ainsi, des stratégies et techniques des cultures mixtes (association maïs-tubercules, maïslégumineuses) sur la même parcelle ont été introduites dans la communauté par les structures d'encadrement du monde rural afin d'éradiquer l'extrême pauvreté, la faim et de renverser la baisse des rendements des maïs sous l'effet du réchauffement climatique. Des études ont montré

\section{MATÉRIEL ET MÉTHODES}

Présentation de la zone d'étude

Situation de zone d'étude: L'étude a été réalisée dans la Commune de Glazoué. D'une superficie de 1750 km², elle est l'une des six Communes que compte le Département des Collines du Bénin. Glazoué est situé entre $7^{\circ} 90^{\prime}$ et $8^{\circ} 30^{\prime}$ latitude Nord et $2^{\circ} 05^{\prime}$ et $2^{\circ} 22^{\prime}$ longitude Est. Elle est limitée au Nord-Ouest par la Commune de Bantè ; au Nord Est par les Communes de Savè et Ouèssè ; au Sud par la Commune de Dassa-Zoumé; au Sud-Ouest par la Commune de que l'association des cultures de maïs avec des légumineuses présente de multiples avantages. Les légumineuses peuvent améliorer la fertilité du sol notamment son statut azoté (Azontondé, 1993, Bado, 2002 ; Roy et al., 2006). Mais d'autres études sont allées à l'antipode de ce qui précède en soulignant que l'association du maïs avec les légumineuses comme: niébé, l'arachide et mucuna est à la baisse de rendement du maïs par rapport à sa culture pure. Abordant dans le même sens les travaux de Coulibaly et al. (2012) ont montré les problèmes de compétition entre les céréales et les légumineuses. Cela se montre par le fait que l'association de la légumineuse au maïs entraîne une baisse de rendement du grain du maïs par rapport à sa culture pure. De ces constats des questionnements se posent : la réduction de la densité et le décalage de la date de semis du niébé seraient-ils une voie pour atténuer la baisse du rendement de maïs? La connaissance de l'impact (positif ou négatif) de l'association des cultures du maïs avec les plantes de la famille Cucurbitaceae (Citrullus) en occurrence Citrullus lanatus (Thunb) communément «goussi» s'avère importante pour mieux raisonner les choix des espèces pouvant faire l'objet de combinaison véritable avec la culture de maïs pour lutter efficacement contre les effets de la variabilité climatique au Bénin. C'est dans ce contexte que s'inscrit la présente étude qui s'intéresse à l'importance de l'association de cultures de maïs et de goussi face à la variabilité climatique.

Savalou (Assognba, 2014). La Commune compte 48 villages administratifs répartis dans dix (10) arrondissements que sont : Aklampa, Assanté, Glazoué, Gomé, Kpakpaza, Magoumi, Sokponta, Ouèdèmè, Thio et Zaffé. Le village Kpakpazoumè (arrondissent de Zaffé) et le village Amangavissa (arrondissement Aklampa) ont abrité les sites expérimentaux. La figure1 présente la commune de Glazoué. 

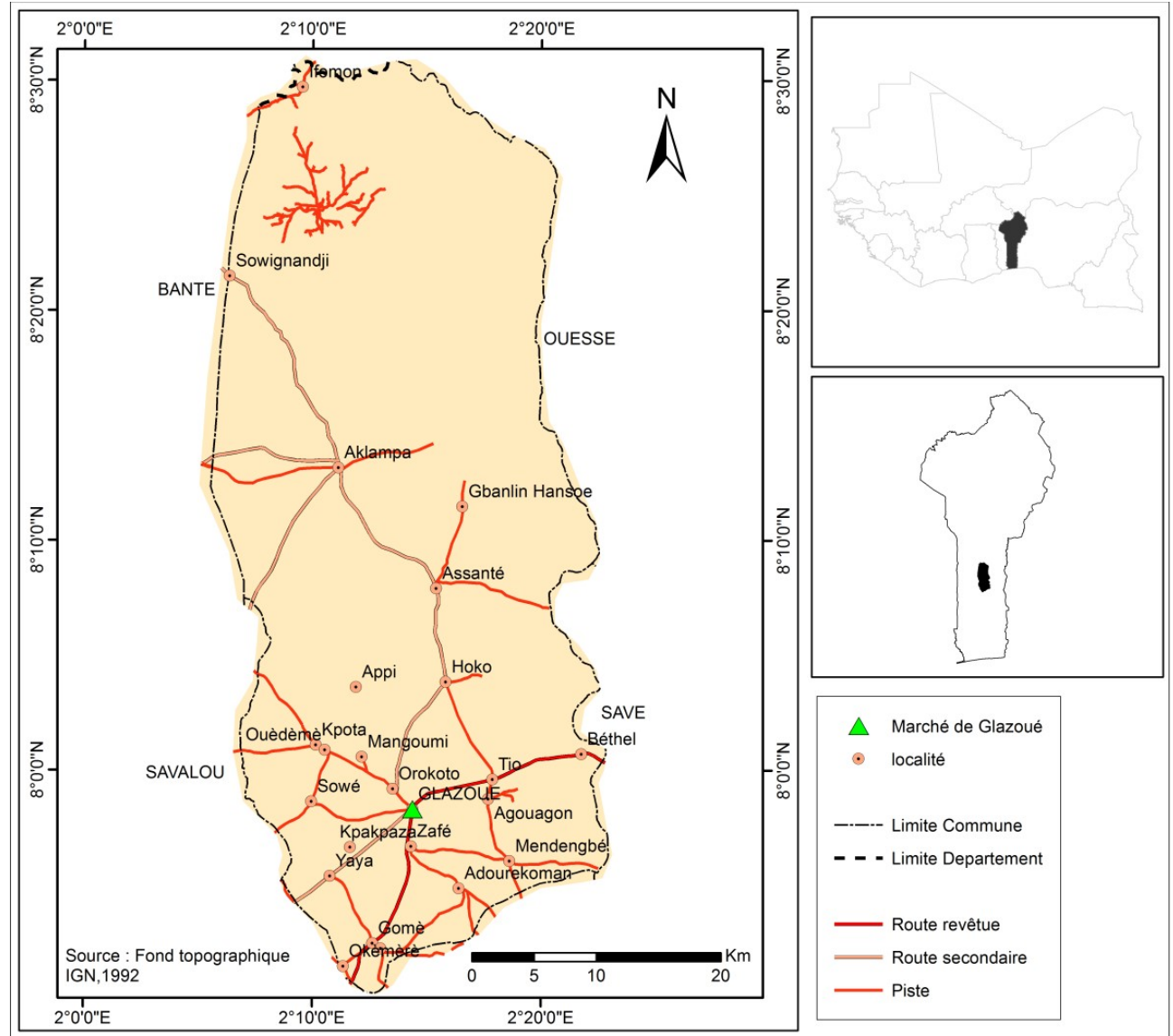

Figure 1 : Localisation de la commune de Glazoué

Climat de la Commune de Glazoué : La Commune de Glazoué est située dans la zone de transition climatique entre le Sub-équatorial et le Soudanien donc caractérisée par un climat Soudano-Guinéen (Balogun et al., 2016). Ce climat est caractérisé par deux saisons pluvieuses dont une petite et deux saisons sèches dont une petite également (Adekambi, 2005 ; Adelman et al., 2008 ; Diagne et al., 2012). Mais depuis quelques années les précipitations de la grande saison des pluies sont irrégulières et un retard s'observe dans leur installation. Par ailleurs, entre la grande saison et la petite saison des pluies la démarcation n'est plus nette. Ces deux saisons semblent se chevaucher, laissant apparaître une période de transition avec une sècheresse d'environ 15 jours. Ainsi la grande saison des pluies s'installe entre Mars et mi-Juillet et la petite saison des pluies d'Août à fin Octobre. La grande saison sèche quant à elle, s'étend de Novembre à
Mars. De Janvier à Mars le temps est souvent sec, ensoleillé avec un régime d'harmattan caractérisé par des matinées et des nuits fraîches $\left(15\right.$ à $\left.20^{\circ} \mathrm{C}\right)$. La température moyenne mensuelle dans la zone d'étude est $28,04^{\circ} \mathrm{C}$ et la moyenne des précipitations annuelles est de $1067 \mathrm{~mm}$, avec 5 mois de saison sèche (Tenté et al., 2011).

Matériel végétal, choix des villages producteurs et dispositif expérimental

Matériel biologique végétatif : Le matériel végétal est constitué des semences de maïs EVDT-str et celles de goussi. Le maïs est semé sur toutes les parcelles aux écartements de 0,80 m en interligne et 0,40 m entre les poquets tandis que le goussi a été semé sept (7) jours avant le semis du maïs aux écartements de $0,80 \mathrm{~m} \mathrm{X} 1$ $\mathrm{m}$. Les photos 1 et 2 présentent les matériels biologiques végétaux (graine de maïs et celles de goussi). 


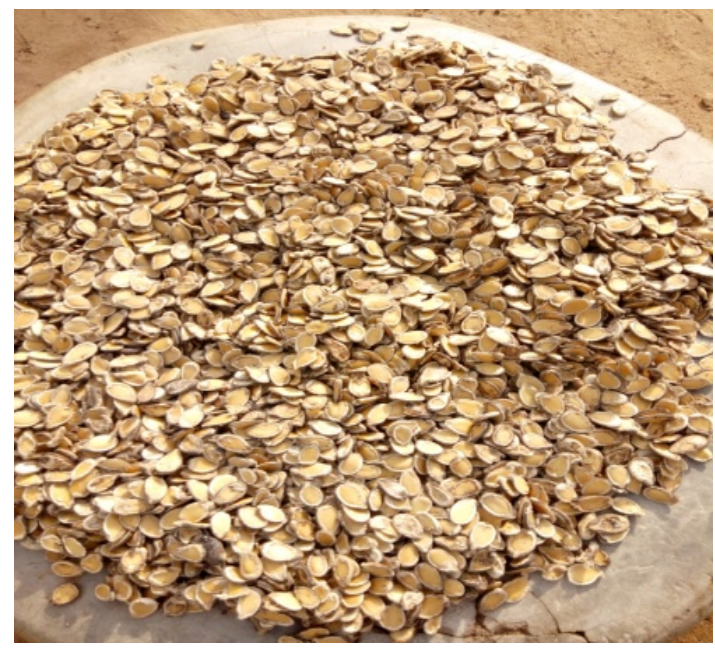

Photo 1. Semence de goussi

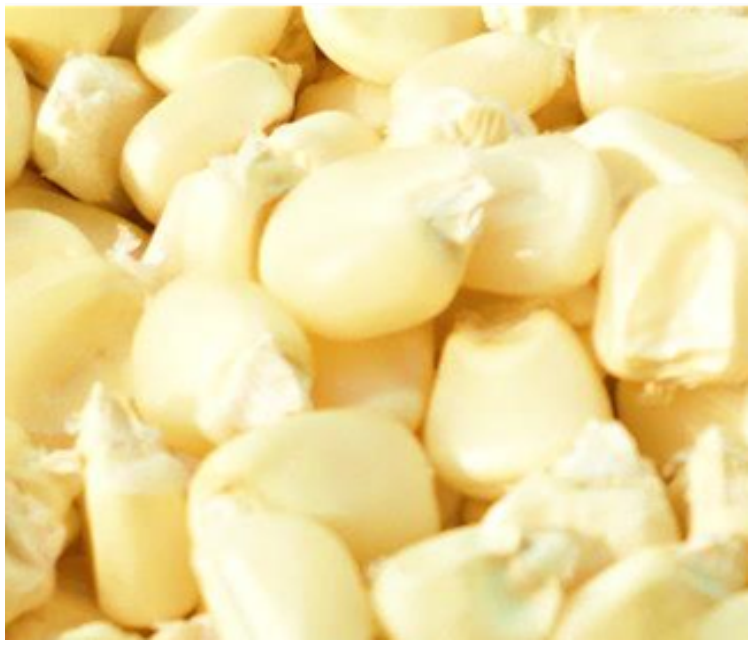

Photo 2. Semence de maïs

Installation du dispositif expérimental : Les essais ont été installés en champs paysans sur un sol ferrugineux tropical dans les villages Kpakpazoumè (Arrondissement Zaffé) et Amangavissa (Arrondissement Aklampa). Le dispositif expérimental est en blocs aléatoires complets $(B A C)$ avec deux répétitions ou blocs. Chaque bloc est subdivisé en deux parcelles élémentaires de $200 \mathrm{~m}^{2}(20 \mathrm{~m} \mathrm{X} 10 \mathrm{~m})$ abritant chacune un traitement. Les traitements sont les suivants : maïs en culture pure (Témoin absolu T) et maïs en association avec le goussi (TesteT 1 ). Les blocs sont séparés de $10 \mathrm{~m}$ et les parcelles à l'intérieur des blocs de $5 \mathrm{~m}$. Au niveau de chaque parcelle, il est mis en place 12 lignes de $20 \mathrm{~m}$ de longueur espacées de $80 \mathrm{~cm}$. La figure 2 présente le dispositif expérimental d'essai.
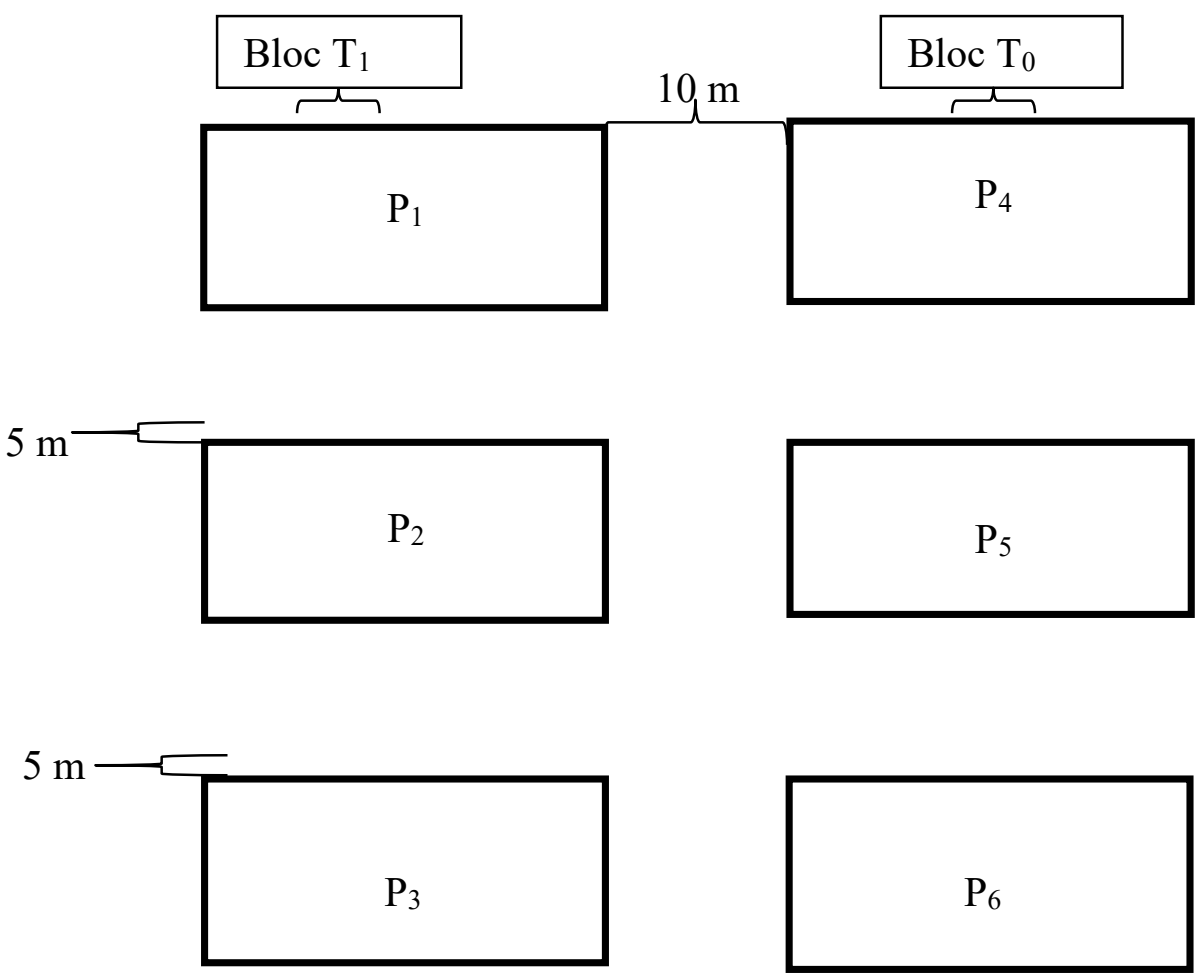

Figure 2 : Dispositif expérimental 


\section{Méthode de collecte des données}

Système de collecte des données : La collecte des données s'est effectuée par trois (3) manières: une phase basée sur des enquêtes auprès des producteurs, une autre par la mesure de taux d'humidité du sol de chaque parcelle et la troisième par des mesures des rendements du maïs et du goussi :

- le suivi des essais dans chacun des deux (2) villages a été fait avec l'aide des producteurs. Une fiche de suivi de l'itinéraire technique a permis l'enregistrement des données relatives aux dates des opérations culturales et à l'installation des cultures, les temps de travaux, les charges et le nombre d'actifs. Les caractéristiques de parcelles telles que l'âge de mise en culture, le type de sol et les précédents culturaux ont été obtenus par enquêtes auprès des producteurs ;

- la fiche de relevé des observations a servi à l'enregistrement des données relatives au rendement du maïs et celui de goussi. Les mesures de rendements ont été effectuées sur des placettes de $1 \mathrm{~m}^{2}$ installées sur les deux diagonales de chaque parcelle. Ces placettes sont au nombre de cinq (5) par parcelle ( $T_{0}$ et $T_{1}$ ). Au total dix (10) plants de maïs ont fait l'objet de mesure par placette. Les épis de maïs et les fruits du goussi ont été récoltés après la collecte des échantillons dans les carrés de rendement;

- une quantité de sol égale à $500 \mathrm{~g}$ a été prélevé sur chacun des blocs dans des boites complètement sèches et pesé à l'aide de la balance et ensuite envoyée au laboratoire pour mesurer le taux d'humidité.

\section{Données climatique : humidité}

Le paramètre climatique mesuré est l'humidité au niveau des sols de tous les essais. Pour se faire, il est prélevé une quantité de $500 \mathrm{~g}$ de sol au niveau de tous les essais de façon périodique. Ces quantités de sol une fois prélevées sont pesées à l'aide de la balance avant d'être acheminé au laboratoire pour mesurer l'humidité.

Données climatiques : La différence de poids obtenu en faisant les $500 \mathrm{~g}$ de terre moins le poids obtenu après que l'échantillon est passé dans l'étuve a été utilisé pour calculé le taux d'humidité de chaque échantillon. Le taux d'humidité a été obtenu en utilisant la formule suivante.

$H_{(\%)}=\frac{500-p_{e}}{500} \times 100$,

avec $p_{e}$ le poids obtenu après que l'échantillon est passé à l'étuve à $105^{\circ} \mathrm{C}$. Ces données d'humidités ont été saisies dans un tableur Excel après chaque collecte pour les neuf observations faites. Ces données d'humidité ont été soumis à un modèle linéaire à effet mixte sur données longitudinales avec le package nlme (Højsgaard et Halekoh, 2016 ; Halekoh et Højsgaard, 2017). Le facteur système de culture a été considéré comme fixe alors que les facteurs mobiles sont: site aléatoire, bloc et parcelles emboitées dans le site. Les moyennes obtenues par structuration de moyennes ont été présenté graphiquement grâce au package Hmisc (Harrell, 2017). II faut noter que toutes les analyses ont été réalisées dans le logiciel $R$ 3.4.2 (R Core Team, 2017).

Données de production: Les paramètres de productivités du maïs ont été mesurés à travers les performances agronomiques. Les variables exprimant les performances agronomiques du maïs sont : le poids d'un épi de maïs (PEP) en gramme (g), nombre de rangé de grains par épis (NRGEP), nombre de grains par épis (NGEP), longueur d'un épi (LEp) en $\mathrm{cm}$, et poids de 100 grains (P100G) en $\mathrm{g}$.

Le poids d'un épi de maïs (PEP) et celui de 100 grains (P100G) a été pesé à l'aide de la balance.

Le nombre de rangé de grains par épis (NRGEP) et le nombre de grains par épis (NGEP) a été évalué par comptage.

La longueur d'un épi (LEp) de maïs a été mesurée par le mètre.

La planche 1 illustre la photo $A$ des épis de maïs à gauche et celle $B$ des grains à droit. 


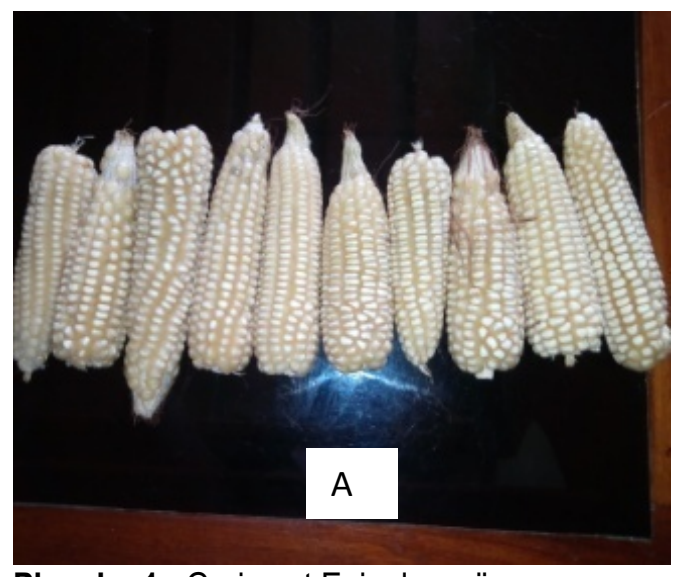

Planche 1 : Grains et Epis de maïs

\section{Analyse statistique des données}

Données de production: Les données sur les performances agronomiques (poids des épis, nombre de rangé par épis, nombre de grains par épis, longueur des épis et le poids de 100 graines) mesurées ont été soumises à un modèle linéaire à effet mixte avec le système de production (Association de maïs et goussi et culture pure de maïs) comme facteur fixe. II est considéré le facteur site comme aléatoire et le bloc comme étant aléatoire emboité dans le site afin que les conclusions de l'expérience puissent être généralisées à d'autres sites au cas où ces derniers ne seront pas significatif. Le package ImerTest (Kuznetsova, et al., 2016) a été utilisé à cet effet. Une structuration des moyennes a été effectuée pour présenter la moyenne et l'erreur type de chacune de ces performances

\section{RESULTATS}

Taux d'humidité du sol au niveau des parcelles cultivées: Le tableau 1 présente les résultats du modèle linéaire à effet mixte sur les données d'humidité du sol. L'analyse de ce tableau révèle que l'humidité du sol au niveau des parcelles de l'association de maïs et goussi est différente de façon significative des parcelles agronomiques par système de production. Ensuite les données sur le rendement en maïs des deux systèmes de production ont été également soumises à un modèle linéaire à effet mixte en considérant encore le facteur système de production comme fixe et les facteurs site et bloc aléatoire emboité dans le site. Une structuration de moyenne a été également faite sur le rendement moyen en maïs des deux systèmes de production. Les données du rendement en goussi du système de production maïs et goussi a été analysé avec le test $t$ de Student après vérification de la normalité des données afin de comparer le rendement des deux sites. Notons que toutes les analyses ont été réalisées dans l'environnement du logiciel $R$ 3.4.2 (R Core Team, 2017).

de la culture pure de maïs $(P<0,001)$. Cette variation significative a été noté pour l'interaction temps et système de culture $(P=0,040<0,05)$. II est aussi noté qu'à l'exception du coefficient intra classe du bloc qui était élevé $(9,28 \%)$, celui du site des parcelles était le plus faible respectivement $1,34 \%$ et $0,52 \%$.

Tableau 1 : Résultats du modèle linéaire à effet mixte sur les données d'humidité du sol

\begin{tabular}{l|l|l|l}
\hline & Coef (se) & t & Prob \\
\hline Intercepte & $61,61(5.51)$ & 11,18 & $<0,001$ \\
Temps & $-0,14(0.03)$ & $-4,96$ & $<0,001$ \\
Système : Culture pure & $14,15(3.07)$ & 4,60 & $<0,001$ \\
Temps : Système : Culture pure & $-0,05(0.09)$ & -133 & 0,040 \\
ICC $_{\text {Site }(\%)}$ & 1,34 & & \\
ICC $_{\text {Block }(\%)}$ & 9,28 & & \\
ICC & 0,52 & & \\
\hline
\end{tabular}


La figure 3 présente les moyennes d'humidité observée au niveau des deux systèmes de culture durant les différents temps de collectes. De l'analyse de ce graphe, il ressort que l'humidité au niveau des parcelles du système de l'association de maïs et goussi est supérieure à celle des parcelles du système de culture pure de maïs et ceci durant tous les temps de collectes. II faut noter que les pics des courbes d'humidité correspondent au temps de collecte juste après une pluie. L'humidité la plus élevée était $75,67 \pm 2,54 \%$ et
$69,50 \pm 2,42 \%$ respectivement pour le système association de maïs et goussi et le système de culture pure de maïs. Ces valeurs ont été observées au 58ème jour après semis. Les taux d'humidités les plus faibles ont été notés vers les 79ème et 86 ème jours qui étaient respectivement de $45,63 \pm 1,13 \%$ et $39,20 \pm 3,27 \%$ pour le système d'association maïs et goussi et le système de culture pure de maïs. Cette baisse de taux d'humidité est due à la réduction du couvert végétal de goussi suite à sa maturité complète.

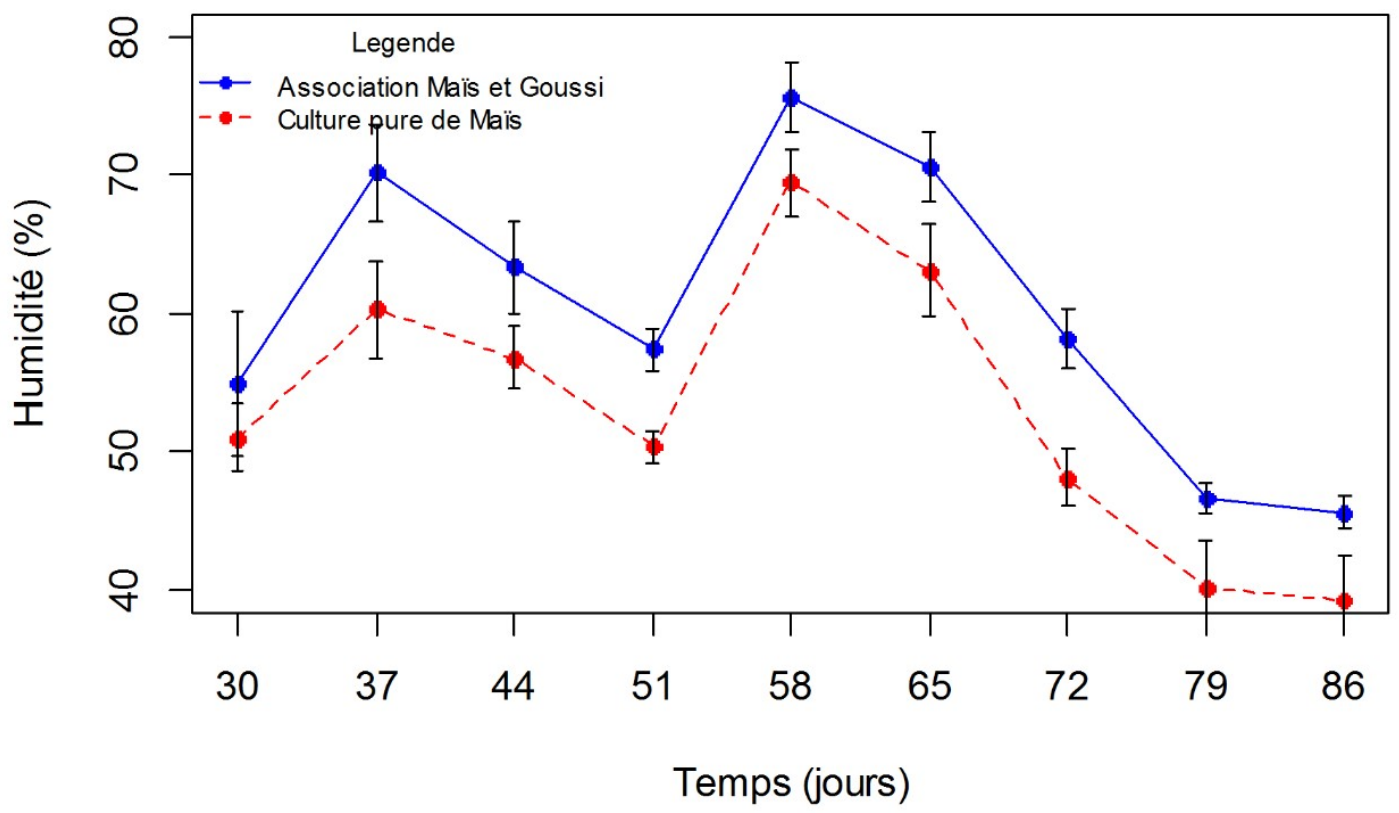

Figure 3 : Moyennes d'humidité observée au niveau des deux systèmes de culture

\section{Les paramètres de production}

Effets des sites et bloc emboités sur les performances agronomiques des épis de maïs : Le tableau 2 présente les résultats du modèle linéaire à effet mixte sur les performances agronomiques. L'analyse de ce tableau montre qu'à l'exception de la longueur des épis (LEp) qui n'a pas varié significativement selon le système de production, tous les autres paramètres (le poids des épis (PEP), le nombre de rangé par épis, le nombre de grains par épis (NGEP) et le poids de 100 graines (P100G) ont variés de manière significative au seuil de $5 \%$ pour le système de production. Les facteurs aléatoires bloc emboités dans site et le facteur aléatoire site n'ont pas eu un effet significatif pour le poids des épis, le nombre de rangé d'épis ( $R^{2}$ conditionnel $=R^{2}$ marginal). Mais on note un effet significatif des facteurs aléatoires bloc et site pour le nombre de grains par épis, la longueur des épis et le poids de 100 graines ( $R^{2}$ conditionnel $>R^{2}$ marginal). II ressort de ces analyses que la performance agronomique du maïs en association avec goussi est meilleure que le maïs en culture pure. Cette bonne performance agronomique dépend des facteurs aléatoires bloc et site. 
Tableau 2 : Résultats du modèle linéaire à effet mixte sur les données de production

\begin{tabular}{lllllll}
\hline & & PEP & NRGEP & NGEP & LOEP & P100G \\
\hline Système de production & $\mathrm{F}$ & $67.68^{\star * *}$ & $5.20^{*}$ & $106.85^{\star * *}$ & 13.65 & $45.33^{*}$ \\
Block : Site & Variance & 0.04 & 0.00 & 0.00 & 0.01 & 4.44 \\
Site & Variance & 0.01 & 0.00 & 0.00 & 0.01 & 1.63 \\
Résidus & Variance & 19.22 & - & - & 0.52 & 49.71 \\
$\mathrm{R}^{2}$ marginal & $(\%)$ & 17.67 & 1.39 & 25.14 & 12.61 & 57.08 \\
$\mathrm{R}^{2}$ conditionnel & $(\%)$ & 17.67 & 1.39 & 29.30 & 17.43 & 61.76 \\
\hline
\end{tabular}

$\mathrm{F}$ : test de Fischer, ${ }^{* * *}$ : significativité à $0.1 \% ;{ }^{* *}$ : significativité à $1 \% ;{ }^{*}$ : significativité à $5 \%$

Moyenne et erreur type performances agronomiques: La figure 4 présente la moyenne et l'erreur type des performances agronomiques pour chaque système de production. Ce graphe montre que le poids des épis était le plus élevé $(155,15 \mathrm{~g} \pm 1,19)$ pour l'association maïs et goussi de même que pour tous les autres paramètres (le nombre de rangés par épis, le nombre de grains par épis et le poids de 100 graines). Autrement dit, le nombre de rangé d'épis $(13,33 \pm 0,04)$, le nombre de grains par épis $(349,21 \pm 1,09)$ et le poids de 100 graines
$(372,12 \mathrm{~g} \pm 0,46)$ étaient plus élevé pour l'association maïs et goussi contre ceux de la culture pure de maïs qui étaient respectivement de 12,38 rangé / épis $\pm 0,12$; 320 grains/épis. $16 \pm 1,82$ et $351,8 \mathrm{~g}$ pour 100 graines $\pm 0,37$. Cela peut s'expliquer par le fait que goussi qui est semé sept (7) jours avant le maïs arrive à couvrir complètement le sol pendant la période sensible (apparition des fleurs mal jusqu'à l'épiaison) où la culture du maïs est exigeante en eau et en éléments fertilisants pour sa production.
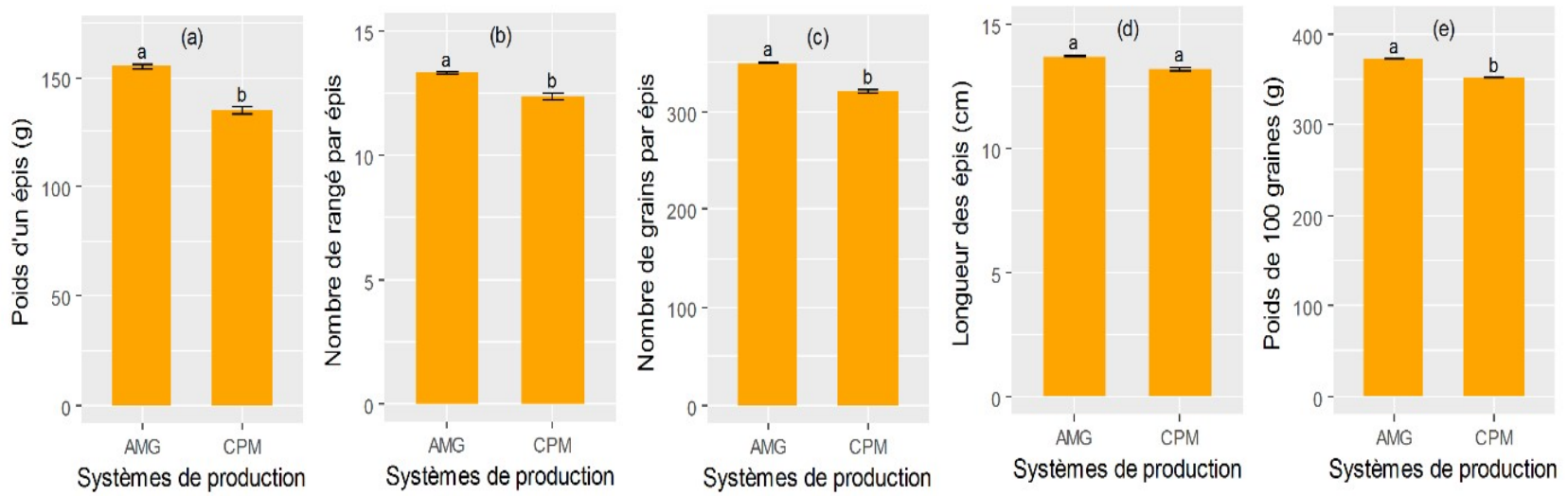

Figure 4. Moyennes des performances agronomiques des systèmes de production (AMG : Association Maïs et Goussi ; CPM : Culture pure de Maïs).

\section{Productivité}

Effets du bloc sur les systèmes de production: Le tableau 3 présente les résultats du modèle linéaire à effet mixte sur le rendement en maïs des systèmes de production. On remarque que Le bloc a eu un effet aléatoire significatif sur le rendement $\left(R^{2}\right.$ conditionnel $>$ $\mathrm{R}^{2}$ marginal).

Tableau 3 : Résultats du modèle linéaire à effet mixte sur le rendement en maïs des systèmes de production

\begin{tabular}{l|l|l|l}
\hline Variantes & Coef (se) & t & Prob. \\
\hline Intercepte & $1728(14.34)$ & 120.53 & $<0.001$ \\
Système : Culture pure & $-38.67(20.28)$ & -1.91 & 0.197 \\
Bloc : Site & 343.3 & & \\
Site & 0.00 & & \\
Résidus & 203.3 & & \\
$\mathrm{R}^{2}$ marginal & 42.72 & & \\
$\mathrm{R}^{2}$ conditionnel & 78.69 & \\
\hline
\end{tabular}


Moyenne de la productivité en maïs pour un hectare par système de production : Le rendement moyen en maïs des deux systèmes de production se présente dans le tableau 4. On note que bien qu'il n'y a pas eu de différences significatives pour le rendement selon les systèmes de production, le système d'association maïs et goussi a présenté le fort rendement (1728 $\mathrm{Kg} / \mathrm{ha} \pm 7,61$ ) contre la culture pure du maïs qui a donné un rendement de $1689 \mathrm{Kg} / \mathrm{ha} \pm 886$. La figure 5 donne la productivité moyenne en maïs des systèmes de production.

Tableau 4 : Rendement moyen en maïs pour chaque système de production

\begin{tabular}{l|l|l}
\hline Systèmes de production & & Production $\mathbf{K g} / \mathbf{h a})$ \\
\hline Association Maïs et Goussi & $\mathrm{m}$ & $1728^{\mathrm{a}}$ \\
& se & 7,61 \\
Culture pure de Maïs & $\mathrm{m}$ & $1689,33^{\mathrm{a}}$ \\
& se & 8,86 \\
\hline
\end{tabular}

$\mathrm{M}=$ moyenne $; \mathrm{se}=$ erreur type

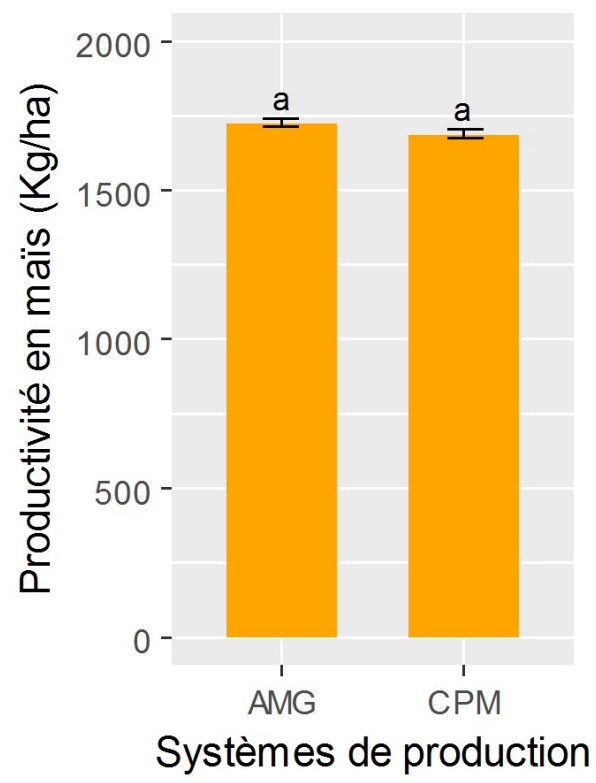

Figure 5. Productivité moyenne en maïs des systèmes de production. (AMG : Association Maïs et Goussi ; CPM : Culture pure de Maïs).

Productivité en Goussi pour un hectare par site de production : Le rendement en goussi obtenu sur les deux sites ont été comparé en utilisant le test de comparaison de deux moyennes et les résultats sont présentés dans le tableau 5. On remarque qu'il n'y a pas eu une différence significative pour le rendement en goussi des deux sites. Les résultats donnent à Amangavissa un rendement en goussi de $803,3 \mathrm{Kg} / \mathrm{ha}$ alors qu'à Kpapkazoumè le rendement a été un peu plus élevé 806,7 Kg/ha.

Tableau 5 : Rendement en goussi par site pour le système d'association Maïs et goussi ( $m=$ moyenne ; se = erreur type)

\begin{tabular}{l|l|l|l|l}
\hline Site & & Production (Kg/ha) & Prob. \\
\hline Amangavissa & & $\mathrm{m}$ & 803,3 & 0,876 \\
& & $\mathrm{se}$ & 6,7 & \\
Kpapkazoumè & $\mathrm{m}$ & 806,7 & \\
& & $\mathrm{se}$ & 18,0 & \\
\hline
\end{tabular}




\section{DISCUSSION}

La diversité des stratégies d'adaptations mises en place par les producteurs montrent une réelle volonté de minimiser les impacts des changements climatiques. La jachère permet d'atténuer les effets négatifs de la pauvreté du sol pour lui redonner vie. Cependant, cette stratégie parait peu efficace pour d'autres qui, préfèrent pratiquer l'association de culture avec les légumineuses. La présente étude qui porte sur l'importance de l'association des cultures de maïs et de goussi face aux variabilités climatiques dans la commune de Glazoué a révélé que le taux d'humidité au niveau des parcelles du système de l'association de Maïs et Goussi est supérieure à celle des parcelles du système de culture pure de maïs et ceci durant tous les temps de collectes. Cette variation significative notée est due à la conservation de l'humidité retenue par le couvert végétal du goussi qui alimente favorablement le maïs pour une bonne performance agronomique. L'expérimentation en milieu paysans présente alors une grande variabilité de production. Cette variabilité qui se traduit par des écarts qui peuvent s'expliquer par les différentes conditions dans l'exploitation agricole (Tittonell et al., 2010 ; Kiba, 2012). L'association des cultures comme stratégies la plus utilisée était également le cas pour les producteurs de maïs (Agossou et al., 2012) et de sorgho (Gbaguidi et al., 2015; Dossou-Aminon et al., 2015). De pareils constat avait été aussi fait sur d'autres association de cultures de maïs/niébé et de maïs/mucuna (Coulibaly et al., 2012a et b). En dehors du taux d'humidité, il est noté qu'à l'exception de la longueur des épis (LEp) qui n'a pas varié significativement selon le système de production, tous les autres paramètres (le poids des épis (PEP), le nombre de rangé par épis, le nombre de grains par épis (NGEP) et le poids de 100 graines (P100G)) ont variés de manière significative au seuil de $5 \%$ pour le système de production. Mais il est noté un effet significatif des facteurs aléatoires bloc et site pour le nombre de grains par épis, la longueur des épis et le poids de 100 graines ( $R^{2}$ conditionnel $>R^{2}$ marginal). La bonne performance du maïs ne dépend pas seulement de l'association maïs/goussi mais également du dispositif. L'analyse des rendements du maïs selon les systèmes de production montre que les meilleurs rendements sont obtenus au niveau de l'association de maïs/goussi. Les performances des associations maïs/cucurbitaceae semblent dépendre principalement des dates de semis, et de la densité de peuplement des deux espèces. L'itinéraire technique qui permet d'obtenir les meilleurs résultats correspond à celui appliqué ordinairement sur la culture pure maïs en dehors du sarclo-buttage qui n'est pas réalisé sur le système d'association de culture à cause du couvert végétal du goussi ce qui réduit automatiquement le coût de production au niveau du système d'association de culture. Les résultats montrent que le taux d'enherbement est faible dans l'association par rapport à la culture pure du maïs. Ces résultats sont en accords avec ceux de Segda et al. (2014), Cesar et al (2004) et Hien (2004) qui ont montré l'influence positive des légumineuses dans la lutte contre les adventices. Pour Hien (2004) cela est lié à la faculté des légumineuses à couvrir rapidement le sol, limitant ainsi l'enherbement et l'émergence des mauvaises herbes. On note également que l'écart de la date de semis des deux espèces mises en association (maïs et goussi) favorise le développement de chacune des cultures et leurs rendements. Cela peut s'expliquer par le fait que le goussi qui est semé 7 jours avant le maïs arrive à couvrir complètement le sol pendant la période sensible (de l'apparition des fleurs mal jusqu'à l'épiaison) où la culture du maïs est exigeante en eau et en éléments fertilisants pour sa production. Nos résultats confirment donc l'importance du couvert végétal de la culture de goussi sur le maintien de l'humidité dans le système d'associations de cultures maïs/cucurbitaceae (goussi). L'analyse des temps de travaux ne révèle pas de différence significative entre la culture pure du maïs et l'association maïs/curcubitaceae (goussi). Ces résultats ne sont pas conformes à ceux obtenus par Renard et Garba, (1989) ; Coulibaly et al. (2012a) et Segda et al. (2014) en milieu réel. Ils avaient montré sans tenir compte des temps de récolte que les associations mais-légumineuses n'ont pas influencé significativement les temps de travaux comparativement à la culture pure. Cela peut s'expliquer par le fait que l'opération de buttage réalisée sur la parcelle de culture pure du maïs a été remplacée par un désherbage. Les résultats sur les rendements montrent que l'association maïs-goussi entraine une hausse de rendement du maïs par rapport à sa culture pure, mais de façon non significative aux seuils de $5 \%$. Le rendement grain du maïs en association (1728 $\mathrm{kg} / \mathrm{ha}$ ) est supérieur à celui obtenu en culture pure (1689 kg/ha). L'association maïs-goussi semble donc se traduire par une amélioration du rendement. Ces résultats pourraient s'expliquer par la compétition pour l'alimentation minérale et hydrique qui est plus accrue pour le fort recouvrement de la surface du sol par les feuilles des curcubitaceae (goussi). Coulibaly et al. 
(2012a) sont parvenus aux résultats contraires pour l'association du maïs et du niébé. Ils avaient enregistré une baisse significative de l'ordre de $23 \%$ de rendement en grains de maïs associé au niébé par rapport à la culture pure de maïs (Dao., 2014). De la même manière, la baisse de rendement en grains de maïs associé avec mucuna (légumineuse) a été montrée déjà par Azontonde (1993) au Bénin qui avait obtenu dans son étude des rendements en grains de maïs de $1300 \mathrm{~kg} / \mathrm{ha}$ en culture pure et de $200 \mathrm{~kg} / \mathrm{ha}$ en

\section{CONCLUSION ET APPLICATION DES RESULTATS}

L'objectif de cette étude était d'évaluer l'effet de l'association de culture de maïs et de goussi sur l'amélioration de la productivité du maïs un contexte d'adaptation aux changements climatiques dans la commune de Glazoué. Les résultats sur les performances agronomiques et économiques montrent que l'insertion de la culture de goussi entraine de différence significative entre les productions de la culture pure du maïs et les associations maïs/goussi quelle que soit la modalité d'association. De même, le goussi semé 7jours avant le maïs, n'induit pas la baisse du rendement en grain de maïs. Les performances économiques des associations culturales ont ainsi été améliorées par rapport à la culture pure du maïs (traitement TO). L'adoption de l'association du maïs

\section{REFERENCES BIBLIOGRAPHIQUES}

Abderrahmani B., Abbou M., Dobbi A. \& Hassini N., 2006. Evénements climatiques Caractérisation de la Sécheresse dans la Région d'Oran. Les risques liés au temps et au climat, 37 .

Aboh A.B., Olaafa M., Doussou-Gbete G.S.O., Dosso A.D. et Djagoun N., 2002. Ingestion volontaire et digestibilité apparente d'une ration à base de la farine de graines de Mucuna pruriens va. utilis complétée de fourrages chez les lapins, Tropicultura, 20(4): 165169.

Adekambi S.A. 2005. Impact de l'adoption des variétés améliorées de riz sur la scolarisation et la santé des enfants au Bénin : cas du département des Collines. Thèse pour l'obtention du diplôme d'Ingénieur Agronome, Université d'Abomey-Calavi (Bénin) 127p

Adelman S., Alderman H., Gilligan D.O \& Lehrer K. 2008. The Impact of Alternative Food for Education Programs on Learning Achievement and Cognitive Development in Northern Uganda. Unpublished manuscript, University of Maryland. World Bank, International Food association avec mucuna. L. Au vu des essais, les résultats ont révélé des contraintes liées aux associations de cultures maïs/cucurbitaceae (goussi). Ces contraintes concernent la pression parasitaire que subi le goussi pendant la deuxième saison pluvieuse (mi-juillet à mi-octobre) ce qui rend difficile, voire impossible l'installation de cette culture pendant cette saison. II s'avère important de trouver des moyens pour contrôler ces parasites.

avec le goussi à une densité normale $(0,80 \mathrm{~m} \times 0,40 \mathrm{~m}$ pour le maïs et $0,80 \mathrm{~m} \times 1 \mathrm{~m}$ pour le goussi) permet d'accroître la production en biomasse dans un contexte d'adaptation aux changements climatiques. Ainsi le goussi pourrait bien s'intégrer dans un système de production intensif. II est donc important d'encourager les paysans à adopter les associations culturales céréales/cucurbitacea alimentaires afin d'assurer la sécurité alimentaire humaine. Pour améliorer de manière significative la production du maïs et le revenu issu de cette culture, les paysans doivent cultiver le maïs avec le goussi à une densité normale. Enfin, cette technologie peut à long terme améliorer la fertilité des sols et la sécurité alimentaire humaine.

Policy Research Institute, and University of British Columbia 43p.

Diagne A., Dontsop-Nguezet P.M., Kinkingninhoun F.M., Alia D., Adégbola P.Y., Coulibaly M., Diawara S., Dibba L., Mahamood N., Mendy M., Ojehomon V.T. \& Wiredu A.N. 2012. The impact of adoption of NERICA rice varieties in West Africa.. 58p.

Agossou D.S.M., Tossou C.R., Vissoh V.P. et Agbossou1 K.E., 2012. Perception des perturbations climatiques, savoirs locaux et stratégies d'adaptation des producteurs agricoles béninois African Crop Science Journal, Vol. 20, Issue Supplément s2, pp. 565 $-588$

Akedrin T.N., N'guessa K., AKE-ASSI E. et AKE S., 2010. Effet de légumineuses herbacées ou subligneuses sur la productivité du maïs. Journal of Animal and plant sciences, 8(2): 953-963.

Assongba Y.F., 2014. Ethnobotanique, Ecologie, Répartition spatiale et statut de conservation 
de Dialium guineense (Willd) (Fabaceae) au Bénin. Thèse de doctorat de l'Université d'Abomey-Calavi (UAC) 250p.

Azontonde A., 1993. Dégradation et restauration des terres de barre (sols ferralitiques faiblement dessaturés argilo-sableux) au Benin. Cah.Ortom.Sér.Pédol., 28(2): 217-226.

Bado B.V., 2002. Rôle des légumineuses sur la fertilité des sols ferrugineux tropicaux des zones guinéenne et soudanienne du Burkina Faso. Thèse de doctorat : Université Laval-Québec, $197 \mathrm{p}$.

Balogun O. B., Ojo S.B. and Olorunfemi M.O. 2016. Characterisation of tectonic lineaments in the central equatorial atlantic region of Africa using bouguer anomaly gravity data. Ife Journal of Science vol. 18, no. 4 ; 931-947

Carsky R.J., Douthwaite B., Manyong V.M., Sanginga N., Schulz S., Vanlauwe B., Diels J., Keatinge J.D.H., 2003. Amélioration de la gestion des sols par l'introduction de légumineuses dans les systèmes céréaliers des savanes africaines. Cahiers Agriculture, $12: 227-233$

César J. et Gouro A., 2004. Les légumineuses fourragères 'herbacées. Production animale en Afrique de l'Ouest. Fiche technique NO 7, CIRDES, Bobo-Dioulasso, 8p.

Cesar J., Ehouinsou M. et Gouro A., 2004. Production fourragère en zone tropicale et conseil aux éleveurs. Rapport Procordel, CIRDES, BoboDioulasso, 47p.

Coulibaly K., 2012a. Analyse des facteurs de variabilité des performances agronomiques et économiques des cultures et de l'évolution de la fertilité des sols dans les systèmes culturaux intégrant les légumineuses en milieu soudanien du Burkina Faso: approche expérimentale chez et par les paysans. Thèse de doctorat: Université Polytechnique de BoboDioulasso (UPB), $165 p$.

Coulibaly K., Vabl E., Autfray P. et Sedogq P.M., 2012b. Performance technico-économique des associations maïs-niébé et maïs-mucuna en situation réelle de culture au Burkina Faso: potentiels et contraintes. Tropicultura. 30 (3): 147-154.

Coulibaly K., VaU E., Autfray P., Bacyé B., Somda., Nacro H.B., Sédogo M.P., 2012. Coconception d'itinéraires techniques de culture pure du niébé (Vigna unguiculata L. Walp.) et du mucuna (Mucuna deeringiana [Bort], Merrill) dans la zone cotonnière Ouest du Burkina Faso : intérêts et limites. Journal of Agriculture and Environment for International Development, 106 (2): 139-155.

Dao Lassina., 2014. Effets de differents modes d'association sur la productivité du maïs (Zea mays L.) et du niébé (Vigna unguiculata L.Walp) en milieu contrôlé. Diplôme de master production végétale. Université Polytechnique de Bobo-dioulasso (UPB), Institut du développement rural (IDR). Pge $39+$ Annexe.

David L., 1987. Cultures traditionnelles de plein champ. Bibliothèque pour le développement durable et les besoins humains essentiels, New Zeland Digital Library. University of Walkato. Peace Corps, 1987: $497 p$.

De Vries M.E., Leffelaar P.A., Sakané N., Bado B.V., Giller K.E., 2011. Adaptability of irrigated rice to temperature change in Sahelianenvironnements. Exp. Agric., 47: 6987.

Dossou-Aminon I, Dansi A, Ahissou H, Cissé, N, Vodouhè $R$, Sanni A. 2015. Climate variability and status of the production and diversity of sorghum (Sorghum bicolor (L.) Moench) in the arid zone of northwest Benin. Genetic Resources and Crop Evolution, 1-21. DOI: 10.1007/s10722-015-0310-y

Dounias 1. 2001. Les systèmes de culture à base de couverture végétale et semis direct en zones tropicales. Centre National d'Etudes Agronomiques des Régions Chaudes (CNEARC)/CIRAD-CA. Etudes et travaux 34p

Dugué, M. J., 2012. Caractérisation des stratégies d'adaptation au changement climatique en agriculture paysanne. Etude de capitalisation réalisée sur les terrains de coopération d'AVSF, 50p.

Ehouinsou M., 2004. Aeschynomene histrix et Stylosanthes scabra seca, deux légumineuses pour améliorer les jachères, complémenter les rations des ruminants et produire des déjections litières pour la fertilisation des sols. Rapport d'atelier de formation sur l'introduction des cultures fourragères dans les systèmes de production d'Afrique de l'ouest, Cotonou (Bénin), décembre

ESPGRN, 1994. La culture fourragère de l'association maïs/dolique. Fiche technique de recherche/Sikasso (Mali), 27 p. 
Garba A.M., 2007. Etude des possibilités d'amélioration des systèmes de production à base de légumineuses alimentaires (niébé-arachide) dans la zone agro-écologique du Fakara, SudOuest du Niger. Mémoire de fin d'études. Gestion des ressources animales et végétales en milieux tropicaux: Université de Liège (Belgique), $72 \mathrm{p}$.

Garba M., Renard C., 1991. Biomass production, yields and water use efficiency in sorne pearl millet/legume cropping systems at Sadore, Niger. In : Soil water balance, in sudanosahelian zone. Publié sous la direction de Sivakumar M.V.K., Wallace J.S., Renard C. et Giroux C. IASH Publication 199. Press, Institute ofHydrology, Walling ford (D.-K.).

Gbaguidi A.A., Faouziath S., Orobiyi A., Dansi M., Akouegninou B. Et Dansi A., 2015. Connaissances endogènes et perceptions paysannes de l'impact des changements climatiques sur la production et la diversité du niébé (Vigna unguiculata (L.) Walp.) et du voandzou (Vigna subterranea (L) Verdc.) au Bénin

Gnangle, P. C., Egah, J., Baco, M. N., Gbemavo, C. D., Kakaï, R. G., \&Sokpon, N., 2012. Perceptions locales du changement climatique et mesures d'adaptation dans la gestion des parcs à karité au Nord-Bénin. International Journal of Biological and Chemical Sciences, 6(1), 136149.

Guyot, F., Richet, P., Courtial, P., \& Gillet, P., 1993. High-temperatureheatcapacity and phase transitions of CaTiO 3 perovskite. Physics and Chemistry of Minerals, 20(3), 141-146.

Halekoh U, Højsgaard S., 2017. pbkrtest: Parametric Bootstrap and Kenward Roger Based Methods for Mixed Model Comparison. R package version 0.4-7, URL https: I/CRAN.Rproject.org/package=pbkrtest.

Harrell Jr F.E., 2017. Hmisc: Harrell Miscellaneous. R package version 4.0-3, URL https: //CRAN.Rproject.org/package=Hmisc

Hiema S.C., 2005. Caractérisation et classification de lignées de maïs (Zea mays $L J$ ). Mémoire de fin d'études, IDRIUPB, $71 \mathrm{p}$.

Hien E., 2004. Dynamique du carbone dans un acrisol ferrique du Centre-Ouest du Burkina Faso: influence des pratiques culturales sur le stock et la qualité de la matière organique. Thèse de doctorat, Ecole Nationale supérieure Agronomique de Montpellier, France, 137p.

Højsgaard S, Halekoh U2016). doBy: Groupwise Statistics, LSmeans, Linear Contrasts, Utilities. $\mathrm{R}$ package version 4.5-15, URL https://CRAN.R-project.org/package=doBy.

Jens B.A., 2006. Meilleures techniques et approches de développement pour l'amélioration de l'agriculture au sahel. Groupe de coordination des zones arides. Université Norvégienne de science de la vie. ICRISAT-Niamey (Niger).

Juste E., Bedoussac L. et Prieur L., 2009. Est-il possible d'améliorer le rendement et la teneur en protéine du blé en Agriculture Biologique au moyen de culture intermédiaire ou de cultures associées? Innovations Agronomiques, 4: 165176.

Juste E., Bedoussac L., Prieur L., 2009. Est-il possible d'améliorer le rendement et la teneur en protéines du blé en Agriculture Biologique au moyen de cultures intermédiaires ou de cultures associées? Innovations Agronomiques (2009) 4: 165-176. Mandret G., 1988. Le niébé dans l'association légumineuse fourragèrecéréales au Sénégal. ISRAILNERV BP 2037 Dakar. $7 \mathrm{p}$.

Kiba D.I., 2012. Diversité des modes de gestion de la fertilité des sols et leurs effets sur la qualité des sols et la production des cultures en zones urbaine, péri-urbaine et rurale au Burkina Faso. Thèse de doctorat unique, Université Polytechnique de Bobo-Dioulasso, Burkina Faso, $172 \mathrm{p}$.

Kuznetsova Alexandra, Brockhofet Per $B$ and Christensen Rune H.B., 2016. Lmer Test Package: Tests in Linear Mixed Effects Models Journal of Statistical Software. December, Volume 82, Issue 13. doi: 10.18637/jss.v082.113, 1-26

Mémento de l'agronome., 2002. Technology \& Engineering. Ministère des affaires étrangères. CIRAD. Montpellier, France, $1692 p$

Mobamed-Saleem M.A., Otsyina RM., 1986. Fodder banks: For pastoralists or farmers. In: Haque, Nnadi L.A., Haque J., 1986. Forage legumecereal systems: improvement of soil fertility and agricultural production with special reference to Sub-Saharan Africa. In Potentials of forage legumes in farming systems of subSaharan Africa. Actes du séminaire tenu au 
CIPEA, Addis Ababa (Ethiopie), du 16 au 19 Septembre 1985: 212-231

Morris, M. L., 2007. Fertilizer use in African agriculture: Lessonslearned and good practice guidelines. World Bank Publications.

Renard C., Garba M., 1989. Millet based cropping systems with forage legumes for improving nutritive value of crop residues in the sahelian zone. $\mathrm{VI}$ congrès international des herbages. Nice (France), 4-11 octobre 1989. Association française pour la production fourragère (France), 837-838.

R Core Team., 2017. A Language and Environment for Statistical Computing. https://www.Rproject.org/. has been cited by the following.

Roy R.N., Finck A., Blair G.J, Tandon H.L.S., 2006. Plant Nutrition for Food Security: a Guide for Integrated Nutrient Management. FAO Fertilizer and Plant Nutrition Bulletins, 16, FAO 107288: Rome; 237-239.

Roy-Marcauley H. et Tabo R. 2015. Les cultures céréalières : riz, maïs, millet, sorgho et blé, document de référence. 37p.

Savadogo M., Somda J., Seynou o. et Nianogo A.J., 2011. Catalogue des bonnes pratiques d'adaptation aux risques climatiques au Burkina Faso. Ouagadougou, Burkina Faso: UICN Burkina Faso, SOp.

Segda Zacharie, Yameogo P. Louis., Mando Abdoulaye., Kazuki Saito., Wopereis Marco C.S. et Sedogo Michel Papaoba., 2014. Le phosphore limite-t-il la production intensive du riz dans la plaine de Bagré au Burkina Faso ? Int. J. Biol. Chem. Sci. 8(6): 2866-2878 DOI : http://dx.doi.org/10.4314/ijbcs.v8i6.43

Tittonell P., Muriuki A, Shepherd K.D., Mugendi D., Kaizzi K.C., Okeyo J., Verchot L., Coe R., Vanlauwe B., 2010. The diversity of rural livelihoods and their influence on soil fertility in agricultural systems of East Africa - A typology of smallholder farms, Agricultural Systems 103, 83-97.

Tente b. Baglo M.A., Dossoumou J.C. et Yédomonhan H., 2011. Impacts des activités humaines sur les ressources forestières dans les terroirs villageois des communes de Glazoué et de Dassa-Zoumè au centre-Bénin. International Journal Biological Chemical Science 5(5): 2022-2030.

Van Dusen, M. E., \& Taylor, J. E. (2005). Missingmarkets and cropdiversity: evidencefrom Mexico. Environment and DevelopmentEconomics, 10(4), 513-531.

Vissoh A. Sylvain et Assongba Yédjanlognon Fautin., 2017. Urbanization, a challenge for family farming in the western periphery of the city of Abomey-Calavi (South Benin) International Journal of Advanced Engineering and Management Research Vol. 2 Issue 6, 21692179

World Bank., 2009. The World Bank annual report 1818 H St NW Washington DC 20433 USA ISB N 978-0-8213-7946-2

Zougmoré., 1996. L'association culturale sorgho-niébé pour prévenir le ruissellement et l'érosion dans le Sahel au Burkina Faso. Disponible sur le Word Wide Web http://www.idrc.callacro/ev31937201-

Zougmoré R., Guillobez S., Kambou N.F., Ouattara K., 2000. Sorghum-cowpea intercropping: an effective technique against runoff and soil erosion in the Sahel (Sana, Burkina Faso). Arid Soit Research \& rehabilitation 14: 329-342. 